\title{
CORRECTION
}

View Article Online

View Journal I View Issue

Check for updates

Cite this: RSC Adv., 2020, 10, 42765

DOI: $10.1039 / d 0 r a 90120 b$

rsc.li/rsc-advances

\section{Correction: Pharmacophore-based approaches in the rational repurposing technique for FDA approved drugs targeting SARS-CoV-2 $\mathrm{M}^{\text {pro }}$}

\author{
Vishal M. Balaramnavar, ${ }^{\star a}$ Khurshid Ahmad, ${ }^{\text {b }}$ Mohd Saeed, ${ }^{\mathrm{c}}$ Irfan Ahmad, de \\ Mehnaz Kamal ${ }^{f}$ and Talha Jawaid ${ }^{g}$ \\ Correction for 'Pharmacophore-based approaches in the rational repurposing technique for FDA approved \\ drugs targeting SARS-CoV-2 M pro' by Vishal M. Balaramnavar et al., RSC Adv., 2020, 10, 40264-40275, DOI: \\ 10.1039/DORA06038K.
}

The authors regret that the name of one of the authors (Talha Jawaid) was shown incorrectly in the original article. The corrected author list is as shown above.

The Royal Society of Chemistry apologises for these errors and any consequent inconvenience to authors and readers.

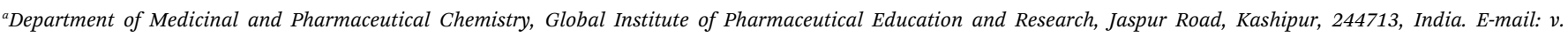
balaramnavar@gmail.com

${ }^{b}$ Department of Medical Biotechnology, Yeungnam University, Gyeongsan 38541, South Korea

${ }^{c}$ Department of Biology College of Sciences, University of Hail, Saudi Arabia

${ }^{d}$ Department of Clinical Laboratory Science, College of Applied Medical Sciences, King Khalid University, Abha, Saudi Arabia

${ }^{e}$ Research Center for Advanced Materials Science, King Khalid University, Abha, Saudi Arabia

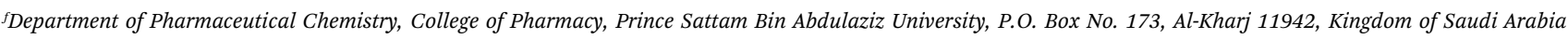

${ }^{g}$ Department of Pharmacology, College of Medicine, Al Imam Mohammad Ibn Saud Islamic University (IMSIU), Riyadh 13317, Saudi Arabia 